Marquette University

e-Publications@Marquette

$1-1-2017$

\title{
Cofacially Arrayed Polyfluorenes: Spontaneous Formation of $\pi$-Stacked Assemblies in the Gas Phase
}

Maxim Vadimovich Ivanov

Marquette University

Neil Reilly

Marquette University

Brandon Uhler

Marquette University

Damian Kokkin

Marquette University

Rajendra Rathore

Marquette University, rajendra.rathore@marquette.edu

See next page for additional authors

Accepted version. The Journal of Physical Chemistry Letters, Vol. 8, No. 21 (2017): 5272-5276. DOI.

(C) 2017 American Chemical Society. Used with permission. 
Authors

Maxim Vadimovich Ivanov, Neil Reilly, Brandon Uhler, Damian Kokkin, Rajendra Rathore, and Scott A. Reid 
Marquette University

\section{e-Publications@Marquette}

\section{Chemistry Faculty Research and Publications/College of Arts and Sciences}

This paper is NOT THE PUBLISHED VERSION; but the author's final, peer-reviewed manuscript. The published version may be accessed by following the link in the citation below.

Journal of Physical Chemistry Letters, Vol. 8, No. 21 (2017): 5271-5276. DOI. This article is (C) American Chemical Society and permission has been granted for this version to appear in ePublications@Marquette. American Chemical Society does not grant permission for this article to be further copied/distributed or hosted elsewhere without the express permission from American Chemical Society.

\section{Cofacially Arrayed Polyfluorenes: Spontaneous Formation of $\pi$-Stacked Assemblies in the Gas Phase}

Maxim V. Ivanov

Department of Chemistry, Marquette University, Milwaukee, WI

Neil Reilly

Department of Chemistry, Marquette University, Milwaukee, WI

Brandon Uhler

Department of Chemistry, Marquette University, Milwaukee, WI

Damian Kokkin

Department of Chemistry, Marquette University, Milwaukee, WI

Rajendra Rathore

Department of Chemistry, Marquette University, Milwaukee, WI

Scott A. Reid

Department of Chemistry, Marquette University, Milwaukee, WI 


\section{Abstract}

\section{Probing $\pi$-stacked fluorene clusters through ionization}
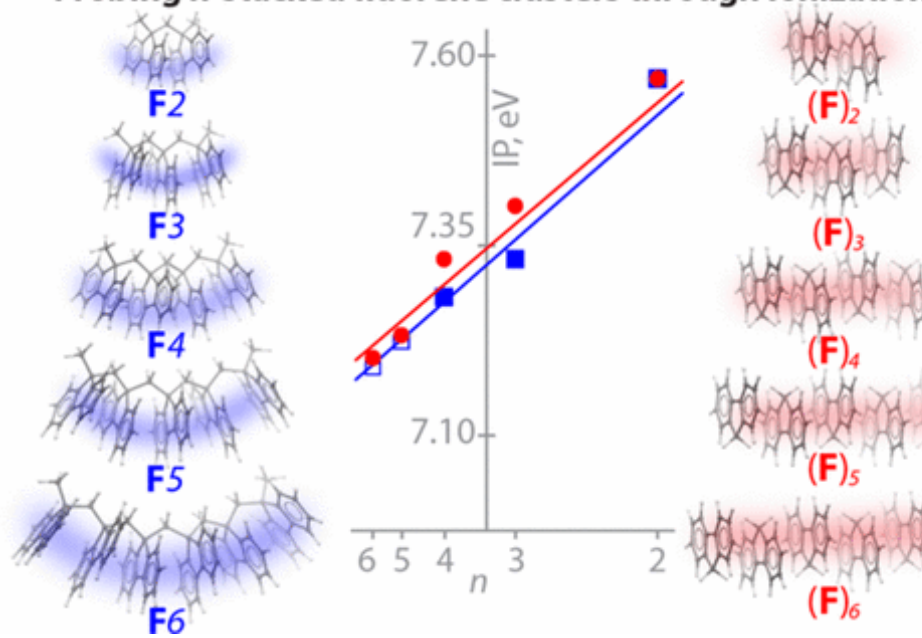

$(\mathbf{F})_{3}$

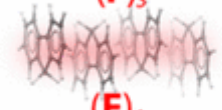

$(\mathbf{F})_{4}$
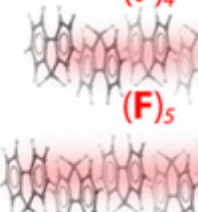

$(\mathbf{F})_{s}$

$(\mathbf{F})_{6}$

Understanding geometrical and size dependencies of through-space charge delocalization in multichromophoric systems is critical to model electron transfer and transport in materials and biomolecules. In this work, we examine the size evolution of hole delocalization in van der Waals clusters of fluorene (i.e., $\left.(\mathbf{F})_{n}\right)$, where a range of geometries are possible, reflecting both $\pi$-stacking and $\mathrm{C}-\mathrm{H} / \pi$ interactions. Using mass-selected two-color resonant two-photon ionization spectroscopy (2CR2PI), we measure electronic spectra and vertical ionization potentials (IPs) in the gas phase. Results are compared with model covalently linked assemblies (denoted $\mathbf{F} n$ ), exhibiting a sterically enforced cofacial (i.e., $\pi$-stacked) orientation of chromophores. For both systems, an inverse size dependence (i.e., $1 / n$ ) of IP vs cluster size is found. Surprisingly, the values for the two sets fall on the same line! This trend is examined via theory, which emphasizes the important role of $\pi$-stacking, and its geometrical dependencies, in the process of hole delocalization in multichromophoric assemblies.

Through-space charge resonance stabilization (CRS) and hole/electron delocalization in cofacially arrayed multichromophoric assemblies are important processes in areas from biochemistry to functional polymeric materials. $\frac{(1-14)}{2}$ The electronic and geometrical requirements for through-space CRS are issues that continue to be debated. Dimer radical cations have been well investigated, both theoretically and experimentally, in light of understanding charge-transport processes in DNA and $\pi$-stacked assemblies and nucleobase oxidative damage. $\frac{(15-20)}{}$ Here, charge stabilization is evidenced in an enhanced reduction in oxidation potential relative to the monomer and the appearance of a charge-transfer resonance transition in the near-infrared region, reflecting incipient covalent bonding. $\frac{(21,22)}{2}$

Studies of through-space CRS in trimers, tetramers, and larger clusters in the condensed phase are hampered by entropic effects. $\stackrel{(23)}{.}$ However, such larger clusters can be readily generated in the gas phase (Figure 1) from condensation in a cold molecular beam. The extent of CRS in these clusters can be selectively probed by measurement of threshold ionization energies using mass-selected ion-yield spectroscopy, $\stackrel{(24-26)}{2}$ which provides the vertical ionization potentials (IPs) from a structure in which the fluorene moieties are arranged for most efficient CRS/electronic coupling. 


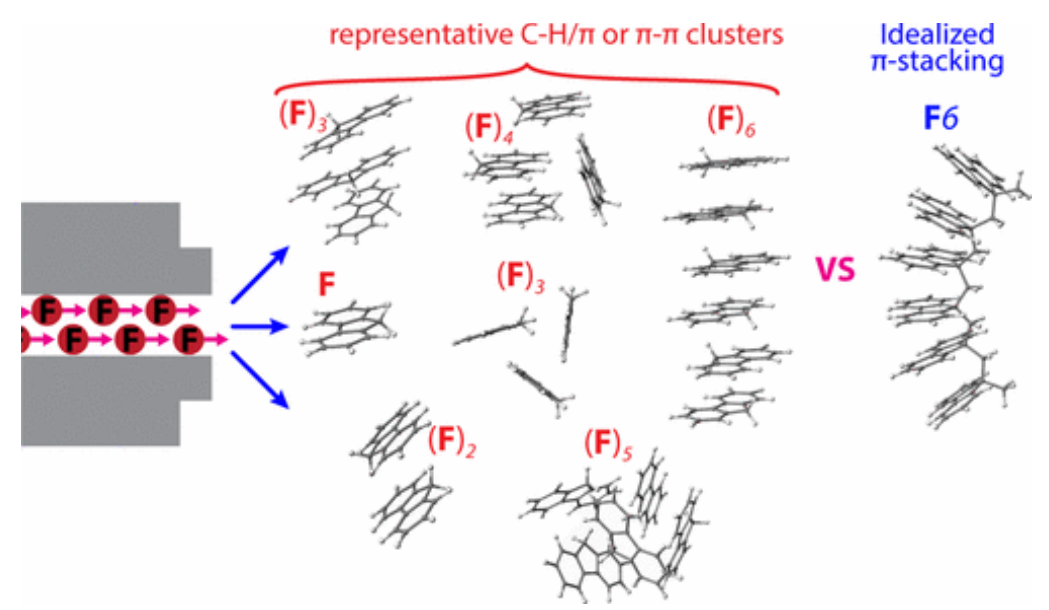

Figure 1. Cartoon representation of the spontaneous formation of the multitude of van der Waals clusters from a supersonic expansion of fluorene and a comparison with a representative covalently linked model hexafluorene F6 in which all fluorenes are cofacially stacked.

Unfortunately, larger clusters inherently exist in a multitude of conformations with varied geometrical arrangement and cofacial overlap and thereby varied CRS/electronic coupling. In order to delineate the structure(s) responsible for the observed IPs from these clusters, herein we make use of a combination of theory and a model set of covalently linked polyfluorenes ${ }^{(27,28)}$ in which all fluorene moieties are cofacially arranged in ideal $\pi$-stacked structures in the gas, liquid, and solid state, thus mimicking the structures that show the lowest ionization energies among various spontaneously formed clusters (Figure 1). ${ }^{(29)}$ While both the van der Waals (i.e., $\left.(\mathbf{F})_{n}\right)$ and covalently linked (i.e., Fn) systems show an inverse length (i.e., $1 / n$ ) dependence of the gas-phase IPs in the measured size range, notably, the trends for $(\mathbf{F})_{n}$ and $\mathbf{F} n$ are very similar, so that the IPs essentially fall on the same line! Below we elucidate the origin of this trend.

Redox properties of covalently linked polyfluorenes (i.e., $\mathrm{F} n, n=1-4$ ) have been previously established ${ }^{(27)}$ both in solution (via cyclic voltammetry) and the gas phase (via photoelectron spectroscopy (PE)). Both adiabatic oxidation potentials $\left(E_{\mathrm{ox}}\right)$ and vertical IPs decrease with an increasing number of fluorene moieties and show a linear dependence when plotted against $1 / n$ (blue squares in Figure 2A).

A
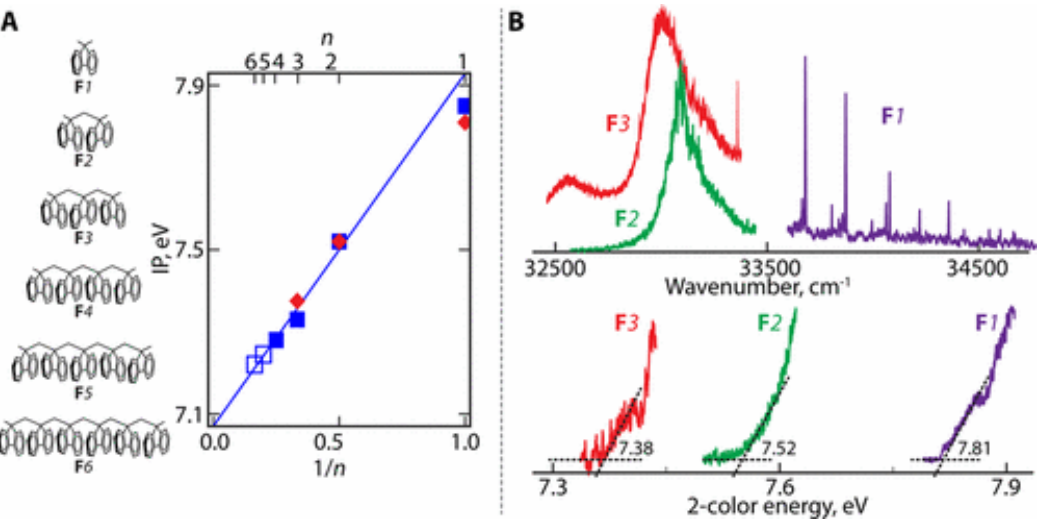
Figure 2. (A) Plot of IPs of Fn obtained from 2 CR2PI (red diamond) and PE 27 (blue filled squares) spectroscopies against the $1 / n$ trend. Empty squares correspond to extrapolated IPs of F5 and F6. (B) Comparison of 2CR2PI spectra and the ion-yield curves of $\mathrm{F} n(n=1-3)$.

Difficulties in obtaining the IPs of higher $\mathbf{F} n$ homologues prompted us to investigate other techniques. For example, in our recent report $\frac{(25)}{2}$ on $\mathbf{F}$, we showed that the same IP as that from PE can be obtained via mass-selected two-color resonant two-photon ionization (2CR2PI) spectroscopy. Both 2CR2PI and laser-induced fluorescence (LIF) spectra of F2 showed a broad unresolved band shifted red by some $1000 \mathrm{~cm}^{-1}$ from the origin band of the monomer $\mathbf{F} 1$ (see Figure 2B, top panel), in agreement with the parallel-displaced cofacial structure in the ground state, which barrierlessly transitions to an excimer sandwich structure in the $S_{1}$ state. This transition is aided by the lowest-frequency torsional mode, which corresponds to a shearing motion, and the inherent Franck-Condon activity and associated vibronic coupling of this mode contribute to the breadth of the experimental spectrum (Figure 2B, top panel).

The broad and largely unresolved structure of the $\mathbf{F} 2$ electronic spectrum is present also in the larger clusters and increases the difficulty of the ion-yield measurements. Nonetheless, mass-selected ion-yield spectra were obtained for $\mathbf{F} 1, \mathbf{F} 2$, and $\mathbf{F} 3$, and their IPs were extracted by measuring the onset of the ion-yield curve (Figure 2B, bottom panel). The determined IP values are identical to our prior $\mathrm{PE}$ measurements and also follow a linear $1 / n$ dependence (Figure $2 \mathrm{~A}$ ).

Mass-selected excitation spectra of the van der Waals clusters $(\mathbf{F})_{n}$ with $n=1-6$ obtained using 2CR2PI are shown in Figure $3 \mathrm{~A}$, top panel. The fluorene dimer, $(\mathbf{F})_{2}$, has been extensively studied, $\left.14,15,30\right)$ and its origin, shifted $217 \mathrm{~cm}^{-1}$ to the red (lower energy) of the monomer, consists of split excitonic components (i.e., $\left.\mathrm{S}_{1} / \mathrm{S}_{2}\right) \frac{(30)}{}$ bearing a complicated substructure. Lifetime broadening is evident, consistent with a ps lifetime that reflects initial movement out of the Franck-Condon region; $\frac{(25)}{a}$ lengthened fluorescence lifetime ( $\sim 60 \mathrm{~ns})$ reflects subsequent excimer formation. Hole-burning spectra indicate that only one conformer contributes to the spectrum, which we have previously identified as a parallel orthogonal $\pi$-stacked structure. $\stackrel{(25)}{ }$ Calculations predict the existence of a second low-lying minimum corresponding to a parallel-displaced $\pi$-stacked structure. ${ }^{(25)}$
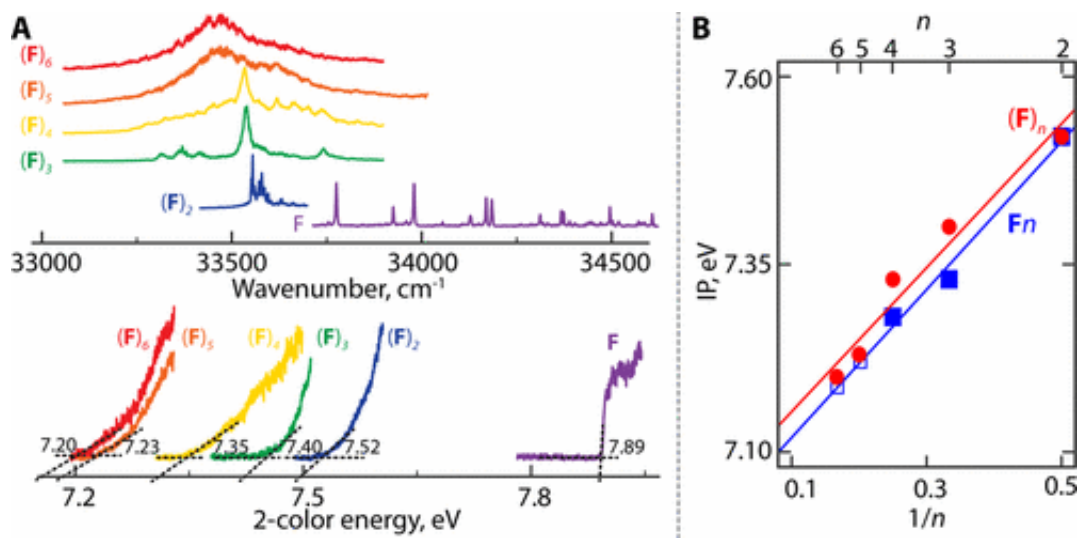

Figure 3. (A) Comparison of 2CR2PI spectra and ion-yield curves of $(\mathbf{F})_{n}(n=1-6)$ clusters. (B) IPs of Fn (blue filled circles) and $(\mathbf{F})_{n}$ (red circles) plotted against the $1 / n$ trend. Empty squares correspond to extrapolated IPs of F5 and F6. 
The evolution of the 2CR2PI spectra with size indicates the presence of multiple conformers for larger clusters (Figure $3 \mathrm{~A}$, top panel). For example, the trimer spectrum shows at least two distinct band systems, one near the position of the dimer origin and a second further red-shifted displaying an extended torsional pattern. We assign these to different conformers, a view supported by the different IPs $(7.40 \mathrm{eV}, 7.44 \mathrm{eV})$ measured following excitation into these two band systems.

Ion yield spectra for the other van der Waals clusters of fluorene are shown in Figure $3 \mathrm{~B}$, bottom panel. The ionization thresholds display a trend of red shift relative to the monomer, with the relative magnitude of the red shift from one cluster to the next decreasing with increasing cluster size. Excluding the monomer, the trend with cluster size indeed follows an approximate $1 / n$ dependence, as shown in Figure $3 \mathrm{~B}$. It must be emphasized that the ion yield spectra of higher clusters will inevitably contain overlapping contributions from multiple conformers (see Figure $3 A$ ). In such a case, our measured ion yield "threshold" will show an onset corresponding to the conformer displaying the smallest IP, a point we examine further below.

The results shown in Figures 2 and $\underline{3}$ clearly show that the IPs of both covalently linked and unlinked (van der Waals) fluorene-based clusters follow a $1 / n$ dependence with increasing cluster size, at least for small $n$. Surprisingly, when plotted together the IP measurements of these two systems fall on the same line, to within our experimental certainty (Figure 3B). To aid in understanding this remarkable trend, we turned to theory. First, molecular dynamics simulations were used to sample the configurational phase space on the ground-state potential energy surface (PES) of $(\mathbf{F})_{n}$ clusters $(n=3-6)$, with subsequent geometry optimizations at the PBE0-D3/def2-SV(P) level, which showed good performance in study of $\pi$ stacked clusters. $\frac{(25)}{}$ Conformational analysis of the obtained $(\mathbf{F})_{n}$ clusters suggests that all conformations can be roughly divided into three categories: fully $\pi$-stacked conformers with either orthogonal, i.e., orthogonal-stacked $(\mathbf{F})_{n}$, or displaced, i.e., displaced-stacked $(\mathbf{F})_{n}$, arrangement of adjacent fluorenes, non- $\pi$-stacked conformers where $\mathrm{C}-\mathrm{H} / \pi$ interactions are prevalent, and hybrid conformations with a combination of $\pi-\pi$ and $\mathrm{C}-\mathrm{H} / \pi$ interactions (Figure $4 \mathrm{~A}$ and Figures S2-S6 in the Supporting Information). Evidencing the importance of $\mathrm{C}-\mathrm{H} / \pi$ interactions, we found that the nonstacked and hybrid conformations are generally most energetically stable on the ground PES, while fully $\pi$-stacked conformers and especially displaced-stacked $(\mathbf{F})_{n}$ lie up to $50.6 \mathrm{~kJ} / \mathrm{mol}$ higher than the global minima structures, depending on the size of the cluster (Table S1 in the Supporting Information).

A
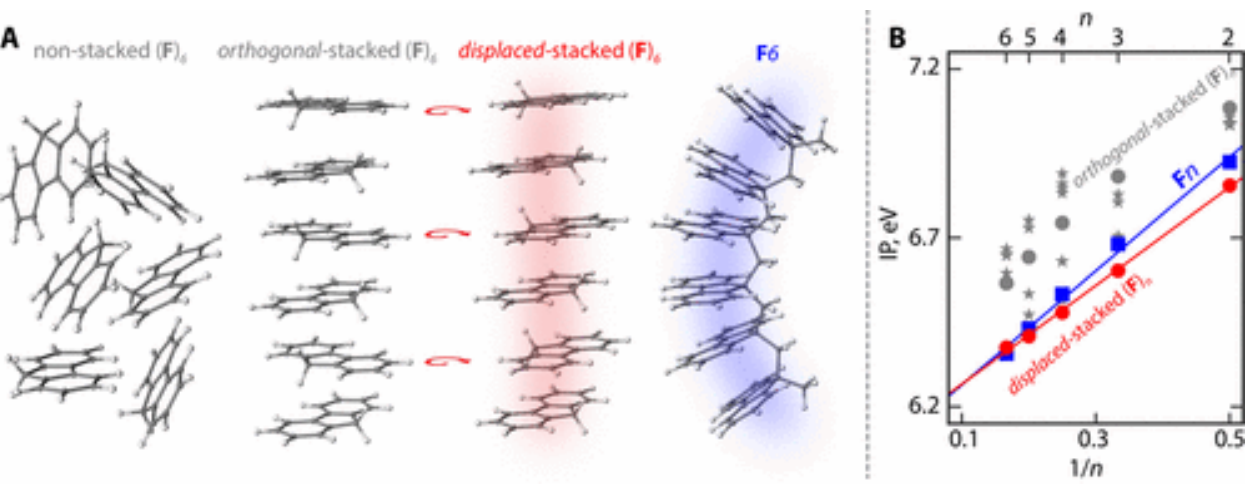

Figure 4. (A) Nonstacked, orthogonal-stacked and displaced-stacked structures of a representative fluorene cluster (F) 6 and a covalently linked structure of F6. Orthogonal- and displaced-stacked clusters differ by the rotation of fluorene unit as indicated. (B) IPs of nonstacked hybrid (gray stars), orthogonal-stacked (gray circles) and displaced-stacked (red circles) fluorene clusters $(\mathbf{F})_{n}$ and covalently linked $\mathbf{F} n$ against the $1 / n$ trend calculated at B1LYP40/6-31G(d). The lines represent linear fits to the data. 
Calculation of the vertical IPs using B1LYP40/6-31G(d), which has been calibrated to reproduce the ionized state of $\pi$-conjugated systems, $\frac{(31,32)}{2}$ shows that the hybrid conformations have the highest IPs, while fully $\pi$-stacked conformers have the lowest IPs, e.g., compare gray stars with red circles in Figure $\underline{4 B}$. This is consistent with the larger overlap and electronic coupling between cofacially array chromophores. Vertical IPs were also calculated for the covalently linked Fn series; Figure 4B displays a comparison of the derived IPs for each set. While the IPs for all orthogonal-stacked $(\mathbf{F})_{n}$ fall on a straight line when plotted vs $1 / n$, these lie uniformly higher than the corresponding IPs of the covalently linked F $n$ (gray circles in Figure 4B). However, the displaced-stacked van der Waals conformers fall exactly on the linear trend for covalently linked $\mathbf{F} n$ (compare blue and red symbols in Figure 4B).

In this work, we have examined the evolution of through-space charge delocalization with increasing size in a series of cofacial covalently linked and unlinked (van der Waals) clusters of fluorene. While their electronic spectra show dramatic differences, understood by the stabilization of a pre-excimeric configuration in the covalently linked system, the measured IPs with cluster size $(n)$ show in each case a $1 / n$ dependence, which fall to within our experimental error on the same line. This trend is well reproduced by theory if displaced, fully $\pi$-stacked van der Waals cluster structures are considered. In contrast, theory predicts that orthogonal $\pi$-stacked and non- $\pi$-stacked structures follow different trends. These displaced, fully $\pi$-stacked van der Waals clusters exhibit similar through-space charge delocalization to a model set of covalently linked fluorenes where cofaciality is enforced by covalent methylene linkages. Thus, our work underscores both the importance of $\pi$-stacking in hole delocalization and the geometrical requirements for hole stabilization in $\pi$-stacked multichromophoric assemblies.

\section{Supporting Information}

The Supporting Information is available free of charge on the ACS Publications website at DOI: 10.1021/acs.jpclett.7b02627.

\section{Acknowledgements}

Support by the National Science Foundation (Grant CHE-1508677) is acknowledged. The calculations were performed on the high-performance computing cluster Père at Marquette University funded by NSF Awards OCI-0923037 and CBET-0521602 and the Extreme Science and Engineering Discovery Environment (XSEDE) funded by NSF (TG-CHE130101).

\section{References}

1. Zabelin, A. A.; Shkuropatova, V. A.; Shkuropatov, A. Y.; Shuvalov, V. A. Temperature Dependence of Light-Induced Absorbance Changes Associated with Chlorophyll Photooxidation in Manganese-Depleted Core Complexes of Photosystem II Biochemistry (Moscow) 2015, 80, 1279- 1287 DOI: 10.1134/S0006297915100089

2. Okubo, T.; Tomo, T.; Sugiura, M.; Noguchi, T. Perturbation of the structure of P680 and the charge distribution on its radical cation in isolated reaction center complexes of photosystem II as revealed by fourier transform infrared spectroscopy Biochemistry 2007, 46, 4390- 4397 DOI: 10.1021/bi700157n 
3. Noguchi, T.; Tomo, T.; Inoue, Y. Fourier Transform Infrared Study of the Cation Radical of P680 in the Photosystem II Reaction Center: Evidence for Charge Delocalization on the Chlorophyll Dimer Biochemistry 1998, 37, 13614- 13625 DOI: 10.1021/bi9812975

4. Lin, S. W.; Imamoto, Y.; Fukada, Y.; Shichida, Y.; Yoshizawa, T.; Mathies, R. A. What Makes Red Visual Pigments Red? A Resonance Raman Microprobe Study of Retinal Chromophore Structure in lodopsin Biochemistry 1994, 33, 2151- 2160 DOI: 10.1021/bi00174a023

5. Malave Osuna, R.; Ruiz Delgado, M. C.; Hernandez, V.; Lopez Navarrete, J. T.; Vercelli, B.; Zotti, G.; Novoa, J. J.; Suzuki, Y.; Yamaguchi, S.; Henssler, J. T.; Matzger, A. J. Oxidation of End-Capped Pentathienoacenes and Characterization of Their Radical Cations Chem. - Eur. J. 2009, 15, 12346- 12361 DOI: 10.1002/chem.200900246

6. Casado, J.; Takimiya, K.; Otsubo, T.; Ramirez, F. J.; Quirante, J. J.; Ortiz, R. P.; Gonzalez, S. R.; Oliva, M. M.; Lopez Navarrete, J. T. Raman Spectroscopy Shows Interchain through Space Charge Delocalization in a Mixed Valence Oligothiophene Cation and in Its $\pi$-Dimeric Biradicaloid Dication J. Am. Chem. Soc. 2008, 130, 14028- 14029 DOI: 10.1021/ja806207j

7. Nakano, T.; Yade, T. Charge Delocalization of Stacked $\pi$-Electron Systems Chem. Lett. 2008, 37, 258-259 DOI: 10.1246/cl.2008.258

8. Grossmann, B.; Heinze, J.; Moll, T.; Palivan, C.; Ivan, S.; Gescheidt, G. Electron Delocalization in One-Electron Oxidized Aniline Oligomers, Paradigms for Polyaniline. A Study by Paramagnetic Resonance in Fluid Solution J. Phys. Chem. B 2004, 108, 4669- 4672 DOI: 10.1021/jp0379042

9. Miyasaka, H.; Khan, S. R.; Itaya, A. Photoinduced electron transfer dynamics in aromatic vinyl polymers and related systems: time-resolved detection of primary events J. Photochem. Photobiol., C 2003, 4, 195- 214 DOI: 10.1016/j.jphotochemrev.2003.09.002

10. Nakano, T.; Yade, T. Synthesis, Structure, and Photophysical and Electrochemical Properties of a $\pi$-Stacked Polymer J. Am. Chem. Soc. 2003, 125, 15474- 15484 DOI: 10.1021/ja037836x

11. Ener, M.; Gray, H.; Winkler, J. Hole Hopping through Tryptophan in Cytochrome P450 Biochemistry 2017, 56, 3531-3538 DOI: 10.1021/acs.biochem.7b00432

12. Gray, H.; Winkler, J. Hole Hopping Through Tyrosine/Tryptophan Chains Protects Proteins from Oxidative Damage Proc. Natl. Acad. Sci. U. S. A. 2015, 112, 10920-10925 DOI: 10.1073/pnas.1512704112

13. Wilson, T. M.; Zeidan, T. A.; Hariharan, M.; Lewis, F. D.; Wasielewski, M. R. Electron Hopping among Cofacially Stacked Perylenediimides Assembled by Using DNA Hairpins Angew. Chem., Int. Ed. 2010, 49, 2385- 2388 DOI: 10.1002/anie.200907339

14. Lewis, F.; Wu, T.; Zhang, Y.; Letsinger, R.; Greenfield, S.; Wasielewski, M. Distance-Dependent Electron Transfer in DNA Hairpins Science 1997, 277, 673- 676 DOI: 10.1126/science.277.5326.673

15. Schumm, S.; Prevost, M.; Garcia-Fresnadillo, D.; Lentzen, O.; Moucheron, C.; Kirsch-De Mesmaeker, A. Influence of the Sequence Dependent lonization Potentials of Guanines on the Luminescence Quenching of Ru-Labeled Oligonucleotides: A Theoretical and Experimental Study J. Phys. Chem. B 2002, 106, 2763- 2768 DOI: 10.1021/jp013185k

16. Sugiyama, H.; Saito, I. Theoretical Studies of GG-Specific Photocleavage of DNA via Electron Transfer: Significant Lowering of Ionization Potential and 5'-Localization of HOMO of Stacked GG Bases in B-Form DNA J. Am. Chem. Soc. 1996, 118, 7063- 7068 DOI: 10.1021/ja9609821

17. Prat, F.; Houk, K.; Foote, C. Effect of Guanine Stacking on the Oxidation of 8-Oxoguanine in BDNA J. Am. Chem. Soc. 1998, 120, 845- 846 DOI: 10.1021/ja972331q 
18. Prat, F.; Houk, K.; Foote, C. Abstr. Pap. Am. Chem. S. 1998, 216, U456

19. Zadorozhnaya, A. A.; Krylov, A. I. Ionization-Induced Structural Changes in Uracil Dimers and Their Spectroscopic Signatures J. Chem. Theory Comput. 2010, 6, 705- 717 DOI: 10.1021/ct900515a

20. Bravaya, K. B.; Kostko, O.; Ahmed, M.; Krylov, A. I. The Effect of $\pi$-Stacking, H-Bonding, and Electrostatic Interactions on the lonization Energies of Nucleic Acid Bases: Adenine-Adenine, Thymine-Thymine, and Adenine-Thymine Dimers Phys. Chem. Chem. Phys. 2010, 12, 2292- 2307 DOI: 10.1039/b919930f

21. Hill, M. G.; Mann, K. R.; Miller, L. L.; Penneau, J. F. Oligothiophene Cation Radical Dimers. An Alternative to Bipolarons in Oxidized Polythiophene J. Am. Chem. Soc. 1992, 114, 2728- 2730 DOI: 10.1021/ja00033a063

22. Hill, M. G.; Penneau, J. F.; Zinger, B.; Mann, K. R.; Miller, L. L. Oligothiophene Cation Radicals. $\pi-$ Dimers as Alternatives to Bipolarons in Oxidized Polythiophenes Chem. Mater. 1992, 4, 11061113 DOI: 10.1021/cm00023a032

23. Navale, T. S.; Thakur, K.; Vyas, V. S.; Wadumethrige, S. H.; Shukla, R.; Lindeman, S. V.; Rathore, R. Charge delocalization in self-assembled mixed-valence aromatic cation radicals Langmuir 2012, 28, 71- 83 DOI: 10.1021/la202611w

24. Uhler, B.; Ivanov, M. V.; Kokkin, D.; Reilly, N.; Rathore, R.; Reid, S. A. Effect of Facial Encumbrance on Excimer Formation and Charge Resonance Stabilization in Model Bichromophoric Assemblies J. Phys. Chem. C 2017, 121, 15580- 15588 DOI: 10.1021/acs.jpcc.7b04255

25. Reilly, N.; Ivanov, M.; Uhler, B.; Talipov, M.; Rathore, R.; Reid, S. A. First Experimental Evidence for the Diverse Requirements of Excimer vs Hole Stabilization in $\pi$-Stacked Assemblies J. Phys. Chem. Lett. 2016, 7, 3042- 3045 DOI: 10.1021/acs.jpclett.6b01201

26. Frey, J.; Holzer, C.; Klopper, W.; Leutwyler, S. Experimental and Theoretical Determination of Dissociation Energies of Dispersion-Dominated Aromatic Molecular Complexes Chem. Rev. 2016, 116, 5614- 5641 DOI: 10.1021/acs.chemrev.5b00652

27. Rathore, R.; Abdelwahed, S. H.; Guzei, I. A. Synthesis, Structure, and Evaluation of the Effect of Multiple Stacking on the Electron-Donor Properties of $\pi$-Stacked Polyfluorenes J. Am. Chem. Soc. 2003, 125, 8712- 8713 DOI: 10.1021/ja035518s

28. Rathore, R.; Chebny, V. J.; Abdelwahed, S. H. A Versatile and Conformationally Adaptable Fluorene-Based Receptor for Efficient Binding of Silver Cation J. Am. Chem. Soc. 2005, 127, 8012- 8013 DOI: 10.1021/ja051935o

29. Vura-Weis, J.; Abdelwahed, S. H.; Shukla, R.; Rathore, R.; Ratner, M. A.; Wasielewski, M. R. Crossover from Single-Step Tunneling to Multistep Hopping for Molecular Triplet Energy Transfer Science 2010, 328, 1547- 1550 DOI: 10.1126/science.1189354

30. Wessel, J.; Beck, S.; Highstrete, C. Excitonic interaction in the fluorene dimer J. Chem. Phys. 1994, 101, 10292- 10302 DOI: 10.1063/1.467909

31. Talipov, M. R.; Boddeda, A.; Timerghazin, Q. K.; Rathore, R. Key role of end-capping groups in optoelectronic properties of poly-p-phenylene cation radicals J. Phys. Chem. C 2014, 118, 21400- 21408 DOI: 10.1021/jp5082752

32. Talipov, M.; Ivanov, M.; Rathore, R. Inclusion of Asymptotic Dependence of Reorganization Energy in the Modified Marcus-Based Multistate Model Accurately Predicts Hole Distribution in Poly-p-phenylene Wires J. Phys. Chem. C 2016, 120, 6402-6408 DOI: 10.1021/acs.jpcc.6b00514 